\title{
Greenberg's conjecture and cyclotomic towers
}

\author{
by \\ David C. Marshall (Austin, TX)
}

1. Greenberg's conjecture. In the late 1950's Iwasawa introduced a powerful technique for studying class groups and unit groups of number fields. Motivated by the theory of curves over finite fields, Iwasawa's theory of $\mathbb{Z}_{p}$-extensions has since become a widely used tool in algebraic number theory, Galois theory, and arithmetic geometry. We describe in this section a conjecture of Greenberg concerning the structure of a classical Iwasawa module, and we mention a Galois-theoretic consequence concerning free pro$p$-extensions of number fields.

Let $K$ be an algebraic number field and $p$ an odd prime. By a multiple $\mathbb{Z}_{p}$-extension $K_{\infty} / K$ we mean a Galois extension with Galois group $\Gamma \simeq \mathbb{Z}_{p}^{d}$ for some positive integer $d$. In what follows we will be particularly interested in two such extensions of $K$ for which we reserve the following notation:

- $K^{\text {cyc }} / K$ denotes the cyclotomic $\mathbb{Z}_{p^{-}}$extension of $K$.

- $\widetilde{K} / K$ denotes the compositum of all $\mathbb{Z}_{p}$-extensions of $K$.

Let $F$ be a finite extension of $K$ contained in $K_{\infty}$, and denote by $A(F)$ the Sylow $p$-subgroup of the ideal class group of $F$. The Galois group of $F / K$ acts on $A(F)$ in the natural way, making $A(F)$ into a $\mathbb{Z}_{p}[\operatorname{Gal}(F / K)]$-module. As $F$ varies over all finite subextensions the $A(F)$ form an inverse system (under norm maps) and we denote by $A$ the inverse limit. The group $A$ then carries a natural structure as a module over the Iwasawa algebra

$$
\mathbb{Z}_{p}[[\Gamma]]:=\lim _{F} \mathbb{Z}_{p}[\operatorname{Gal}(F / K)]
$$

It is common to study $A$ by identifying the $A(F)$ with Galois groups as follows. By class field theory, the group $A(F)$ is isomorphic to the Galois group, $X_{F}$, of the maximal abelian unramified $p$-extension of $F$ (the $p$-Hilbert class field of $F$ ). The isomorphism respects the Galois module structure, the action of $\operatorname{Gal}(F / K)$ on $X_{F}$ being inner automorphism. The $X_{F}$ form an inverse system (the maps being given by restriction of automorphisms)

2000 Mathematics Subject Classification: Primary 11R23; Secondary 11R18, 11R32. 
and the limit $X$ is the Galois group of the maximal abelian unramified pro- $p$-extension of $K_{\infty}$. So $X \simeq A$.

The Iwasawa algebra $\mathbb{Z}_{p}[[\Gamma]]$ is non-canonically isomorphic to the power series ring

$$
\Lambda:=\mathbb{Z}_{p}\left[\left[T_{1}, \ldots, T_{d}\right]\right]
$$

where topological generators $\gamma_{i}$ of $\Gamma$ are sent to $1+T_{i}$. So the $\mathbb{Z}_{p}[[\Gamma]]$ module structure of $A$ is studied via the $\Lambda$-module structure of $X$ (noting that $\left.T_{i} x=x^{\gamma_{i}-1}\right)$.

For $K_{\infty} / K$ any multiple $\mathbb{Z}_{p}$-extension Greenberg [3, Theorem 1] has shown $X$ to be a finitely generated torsion $\Lambda$-module. In particular, the annihilator of $X, \operatorname{Ann}_{\Lambda}(X)$, is non-trivial. Traditionally, annihilators of classical Iwasawa modules have been of much interest. The main conjecture of Iwasawa theory gives the factors of the annihilator of $X$ for the cyclotomic $\mathbb{Z}_{p}$-extension of a number field $K$ as essentially the $p$-adic $L$-functions attached to $K$. There is also a two variable main conjecture for certain $\mathbb{Z}_{p}^{2}$-extensions arising from the theory of elliptic curves.

Greenberg [5, Conjecture 3.4] has conjectured that for the cyclotomic $\mathbb{Z}_{p}$-extension $K^{\text {cyc }} / K$ of a totally real field $K$, the module $X$ is finite. If a totally real field $K$ satisfies Leopoldt's conjecture the extensions $K^{\text {cyc }}$ and $\widetilde{K}$ coincide (i.e. $K$ has only one $\mathbb{Z}_{p}$-extension). Furthermore, when $\Lambda=$ $\mathbb{Z}_{p}[[T]]$ it can be shown that a module being finite is equivalent to having an annihilator of height at least 2 . With this in mind the above conjecture is a special case of the more general conjecture [5, Conjecture 3.5]:

CONJECTURE 1 . Let $K$ be any number field and $\widetilde{K}$ the compositum of all $\mathbb{Z}_{p}$-extensions of $K$. Then $\operatorname{Ann}_{\Lambda}(X)$ has height at least 2 .

A $\Lambda$-module whose annihilator has height at least 2 is said to be pseudonull, and we will refer to Conjecture 1 above as Greenberg's conjecture, or just the pseudo-null conjecture.

The point of this note is two-fold. First, we prove a "going-up" theorem for the pseudo-null conjecture. Namely, if $K$ is a number field, and $F$ is a finite extension of $K$ in $\widetilde{K}$, we give conditions under which Greenberg's conjecture for $K$ implies Greenberg's conjecture for $F$ (Theorem 6). The result is an exercise in utilizing several equivalent formulations of the conjecture. Versions of these formulations have appeared in Lannuzel and Nguyen Quang Do [9, Theorem 4.4] as well as in work of McCallum [11] and this author [10]. Secondly, as an application of the result, we consider the example $K=\mathbb{Q}\left(\zeta_{p}\right)$ and $F=\mathbb{Q}\left(\zeta_{p^{n}}\right)$. We verify the conjecture for a certain class of such $K$ 's, implying the conjecture for each field in the corresponding $\mathbb{Z}_{p}$-tower.

The key argument in both results is reduced to a capitulation problem, namely the need for a set of ideals, or ideal classes, to become principal when 
extended to an appropriate field. For the "going-up" result, the resolution of this problem is provided by an equivalent form of the conjecture, stating that all ideal classes capitulate in $\widetilde{K}$. In verifying the conjecture for $\mathbb{Q}\left(\zeta_{p}\right)$ capitulation is obtained by more direct means. We state our second result here.

Let $K=\mathbb{Q}\left(\zeta_{p}\right), E=\mathcal{O}_{K}^{\times}$and $U=\mathcal{O}_{K_{\pi}}^{\times}$, where $\pi$ is the unique prime of $K$ above $p$. Denote by $\bar{E}$ the closure of $E$ in $U$. We denote by $\lambda_{p}$ the Iwasawa lambda invariant of the cyclotomic $\mathbb{Z}_{p}$-extension of $\mathbb{Q}\left(\zeta_{p}\right)$. Let $v_{p}$ denote the $p$-adic valuation. In Section 4 we prove

THEOREM 1. Suppose $K=\mathbb{Q}\left(\zeta_{p}\right)$ satisfies the following conditions:

(1) Vandiver's conjecture.

(2) $\lambda_{p}=1$.

(3) $v_{p}\left(\left|(U / \bar{E})\left[p^{\infty}\right]\right|\right) \leq v_{p}(|A(K)|)$.

Then for all $n \geq 1$ the pseudo-null conjecture holds for $\mathbb{Q}\left(\zeta_{p^{n}}\right)$.

We mention here one Galois-theoretic consequence of the pseudo-null conjecture for cyclotomic fields. The existence of free pro- $p$-extensions (Galois extensions with Galois group a free pro- $p$-group) has been the subject of much study. See for example the list of known results in [15]. Let $K=\mathbb{Q}\left(\zeta_{p^{n}}\right)$ for some $n>0$, and let $\Omega_{K}$ denote the maximal pro- $p$-extension of $K$ which is unramified at all primes not dividing $p$. Let $\mathcal{G}_{K}$ denote the Galois group.

Since free pro- $p$-extensions are unramified outside $p$, such extensions of $K$ are contained in $\Omega_{K}$. We will see that $\mathcal{G}_{K}$ is a free pro- $p$-group exactly when $p$ is a regular prime (since the number of relations defining $\mathcal{G}_{K}$ is equal to the $p$-rank of the class group of $K)$. When $p$ is an irregular prime the group $\mathcal{G}_{K}$ is not free, but we may look for free pro- $p$-quotients. Let $r_{2}$ denote the number of complex places of $K$. Then Leopoldt's conjecture predicts $r_{2}+1$ independent $\mathbb{Z}_{p}$-extensions of $K$, and so the maximal rank of a free pro- $p$-extension of $K$ is bounded above by $r_{2}+1$. The following is proved in [9], as well as in [11]:

TheOREM 2. Suppose that $K=\mathbb{Q}\left(\zeta_{p^{n}}\right)$ satisfies Greenberg's conjecture. Then $\mathcal{G}_{K}$ has a free pro-p-quotient of rank $r_{2}+1$ if and only if $p$ is regular.

We give here a brief outline of the paper. In Section 2, we introduce several auxiliary $\Lambda$-modules and Galois groups needed for the later study. Theorem 3 and Lemma 1 are the key results of this section, implying a sufficient condition for a standard Iwasawa module to be torsion free (Corollary 1). In Section 3 we recall and provide several equivalent formulations of Greenberg's pseudo-null conjecture, and we state and prove one of our main results (the "going-up" theorem). Finally, in Section 4 we turn to the example furnished by cyclotomic fields, proving Theorem 1 above. 
Acknowledgements. This work is an outgrowth of the authors Ph.D. thesis and he would like to thank his advisor Bill McCallum, as well as Ralph Greenberg and Manfred Kolster for useful conversations and comments. This work was partially supported by NSF VIGRE grant 9977116.

2. Auxiliary modules. For a number field $K$ and a prime number $p$, we call a field extension of $K$-ramified if it is unramified at all primes of $K$ not dividing $p$. We fix the following notation:

The fields:

$\Omega_{K} \quad$ the maximal pro- $p, p$-ramified extension of $K$,

$\widetilde{K} \quad$ the compositum of all $\mathbb{Z}_{p}$-extensions of $K$,

$L_{\infty} \quad$ the maximal abelian unramified pro-p-extension of $\widetilde{K}$,

$M_{\infty} \quad$ the maximal abelian $p$-ramified pro- $p$-extension of $\widetilde{K}$,

$N_{\infty} \quad$ the extension of $\widetilde{K}$ generated by $p$-power roots of $p$-units of $\widetilde{K}$.

The Galois groups:

$\mathcal{G}_{K}$ the Galois group of $\Omega_{K} / K$,

$\Gamma \quad$ the Galois group of $\widetilde{K} / K$,

$X$ the Galois group of $L_{\infty} / \widetilde{K}$,

$Y \quad$ the Galois group of $M_{\infty} / \widetilde{K}$,

$Y^{\prime} \quad$ the Galois group of $N_{\infty} / \widetilde{K}$.

The Galois groups $Y$ and $Y^{\prime}$ carry an action of $\Gamma$ via conjugation, just as $X$, making them into $\Lambda$-modules. We shall see that for certain base fields $K$, the pseudo-null conjecture may be formulated in terms of the $\Lambda$-module structure of $Y$ (in particular, that $Y$ is $\Lambda$-torsion free). The module $Y$ is known to be finitely generated, and, for $K / \mathbb{Q}$ abelian, have $\Lambda$-rank equal to $r_{2}$, where $r_{2}$ denotes the number of complex places of $K$ (see [4]). For a $\Lambda$-module $M$ we write $\operatorname{Tor}_{\Lambda}(M)$ for the $\Lambda$-torsion submodule. The following result is due to McCallum.

TheOREM 3 ([11, Theorem 3]). Suppose there is only one prime of $K$ above $p$, and $\widetilde{K}$ contains all p-power roots of unity. Then $\operatorname{Tor}_{\Lambda}\left(Y^{\prime}\right)=0$.

REMARK 1. The proof of this result involves a detailed analysis of the filtration

$$
E_{F}^{u} \subset E_{F}^{n} \subset E_{F}^{\text {loc }} \subset E_{F},
$$

where $E_{F}$ denotes the units $\mathcal{O}_{F}[1 / p]^{\times}$of a finite extension $F$ of $K$ in $\widetilde{K}$, 
and the superscripts denote certain classes of universal norms (see Section 4 of [11] for the precise definitions). The torsion submodule of $Y^{\prime}$ is contained in the kernel of a surjective map of Galois groups. The Pontryagin dual of this kernel is $\lim _{F}\left(E_{F} / E_{F}^{u}\right) \otimes \mathbb{Q}_{p} / \mathbb{Z}_{p}$, and is shown to be zero by considering each graded factor from the filtration.

REMARK 2. In particular, the result tells us $\operatorname{Tor}_{\Lambda}(Y)$ fixes the field $N_{\infty}$. This observation, combined with Lemma 1 below, gives our approach to verifying the pseudo-null conjecture.

The group $\mathcal{G}_{K}$ has a minimal free presentation

$$
1 \rightarrow R \rightarrow F_{g} \rightarrow \mathcal{G}_{K} \rightarrow 1,
$$

where $F_{g}$ is the free pro-p-group on $g$ generators and $R$ is the normal closure of a finitely generated subgroup (the group of relations for $\mathcal{G}_{K}$ ). Denote by $s$ the minimal number of (topological) generators of $R$. The numbers $g$ and $s$ are equal to the $\mathbb{F}_{p}$-dimensions of $H^{i}\left(\mathcal{G}_{K}, \mathbb{Z} / p \mathbb{Z}\right), i=1,2$, respectively (see Chapter 4 of [13]).

Let $\mathcal{G}_{K}^{\text {ab }}$ denote the maximal abelian quotient of $\mathcal{G}_{K}$, and $M_{K}$ the maximal abelian $p$-ramified pro- $p$-extension of $K$ (so $\mathcal{G}_{K}^{\text {ab }}=\operatorname{Gal}\left(M_{K} / K\right)$ ). The field $M_{K}$ is an abelian, $p$-ramified extension of $\widetilde{K}$ (the Galois group of $M_{K} / \widetilde{K}$ is just the torsion subgroup of $\mathcal{G}_{K}^{\mathrm{ab}}$ ), and so is contained in the field $M_{\infty}$. Hence we have a natural map from $Y$ to $\mathcal{G}_{K}^{\text {ab }}$ given by restriction of automorphisms. We refer the reader to [11] for a proof of the following.

Lemma 1 ([11, Lemma 24]). Suppose $K$ satisfies Leopoldt's conjecture. If $\mathcal{G}_{K}$ is a one-relator group (i.e. $s=1$ ), then the map $\operatorname{Tor}_{\Lambda}(Y) \rightarrow \mathcal{G}_{K}^{\mathrm{ab}}$ is the zero map if and only if $\operatorname{Tor}_{\Lambda}(Y)=0$.

The following is an immediate consequence of Theorem 3 and Lemma 1 :

CoRollary 1. If $K$ is a number field satisfying the hypotheses of Theorem 3 and Lemma 1, then

$$
M_{K} \subset N_{\infty} \text { implies } \operatorname{Tor}_{\Lambda}(Y)=0 .
$$

3. Equivalent formulations. We have introduced the natural Iwasawa modules $X$ and $Y$ in the last section. The Galois action on each of the $X_{F}$ is also compatible with regard to extensions of ideal classes, so we may form the $\Lambda$-module $\lim _{F} X_{F}$ as well. Recall that the groups $\operatorname{Ext}_{\Lambda}^{i}(\cdot, \Lambda)$ are the right derived functors of $\operatorname{Hom}_{\Lambda}(\cdot, \Lambda)$.

THEOREM 4. Let $p$ be an odd prime and let $K$ be a number field with a unique prime above $p$. Then $\operatorname{Ext}_{\Lambda}^{1}(X, \Lambda)$ is the Pontryagin dual of $\lim _{F} X_{F}$, where the $F$ vary over the finite extensions of $K$ in $\widetilde{K}$. 
Proof. Let $\mathfrak{m}$ denote the unique maximal ideal of $\Lambda=\mathbb{Z}_{p}\left[\left[T_{1}, \ldots, T_{r}\right]\right]$, and define

$$
\omega_{n}\left(T_{i}\right)=\left(1+T_{i}\right)^{p^{n}}-1 .
$$

The result is obtained by establishing the isomorphism

$$
H_{\mathfrak{m}}^{r}(X) \simeq \underset{F}{\lim _{F}} X_{F},
$$

where $H_{\mathfrak{m}}^{i}(X)$ denotes Grothendieck's local cohomology relative to the $\mathfrak{m}$ primary sequences

$$
\mathbf{x}_{n}=\left(p^{n}, \omega_{n}\left(T_{1}\right), \ldots, \omega_{n}\left(T_{r}\right)\right)
$$

The desired result is then a consequence of (a version of) Grothendieck's local duality; namely

$$
\operatorname{Ext}_{\Lambda}^{N-i}(X, \Lambda) \simeq \operatorname{Hom}_{\mathbb{Z}_{p}}\left(H_{\mathfrak{m}}^{i}(X), \mathbb{Q} / \mathbb{Z}\right),
$$

where $N$ denotes the length of the m-primary sequence. A good reference for this material is Chapter 3 of [1].

The details establishing (2) can be found in Theorem 8 of [11], where McCallum proves a similar result for the Galois group $X^{\prime}$ of the maximal abelian unramified pro- $p$-extension of $\widetilde{K}$ in which all primes dividing $p$ are completely decomposed. The proof translates easily to this case, simply by replacing the decomposition group with inertia.

Let $\mu_{n}$ denote the group of $n$th roots of unity. As above, we let $X_{F}^{\prime}$ denote the Galois group of the maximal abelian unramified extension of $F$ in which all primes dividing $p$ are completely decomposed. We write $X^{\prime}$ for $X_{\widetilde{K}}^{\prime}$.

TheOREM 5. Let $p>5$ be a prime and suppose $\mu_{p}$ is in $K$. If $K$ has a unique prime ideal $\wp$ dividing $p$, then the following are equivalent:

(a) $X$ is pseudo-null,

(b) $X^{\prime}$ is pseudo-null,

(c) $\operatorname{Tor}_{\Lambda}(Y)=0$,

(d) $\lim _{F} X_{F}^{\prime}=0$,

(e) $\underline{\lim }_{F} X_{F}=0$,

where the fields $F$ vary over all finite extensions of $K$ in $\widetilde{K}$.

Proof. (a) $\Leftrightarrow(\mathrm{b})$. Recall $\Gamma=\operatorname{Gal}(\widetilde{K} / K)$. We let $\Gamma_{\wp}$ denote the decomposition group of $\wp$ in $\Gamma$, and let $\Lambda_{\wp}=\mathbb{Z}_{p}\left[\left[\Gamma / \Gamma_{\wp}\right]\right]$. There is a natural surjection

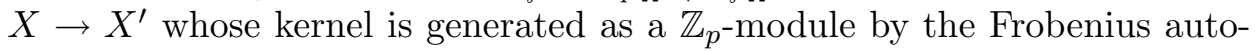
morphisms corresponding to the primes above $p$, and therefore is finitely generated as a module over $\Lambda_{\wp}$. As a $\Lambda$-module, the annihilator of $\Lambda_{\wp}$ has height equal to the $\mathbb{Z}_{p}$-rank of $\Gamma_{\wp}$ (this is just the augmentation ideal in $\left.\mathbb{Z}_{p}\left[\left[\Gamma_{p}\right]\right]\right)$. Since there is only one prime of $K$ above $p$, its decomposition 
group has finite index in $\Gamma$, and therefore our assumption on $p$ makes $\Lambda_{\wp}$ pseudo-null. Therefore the kernel of the surjection $X \rightarrow X^{\prime}$ is pseudo-null, and $X$ and $X^{\prime}$ are pseudo-isomorphic.

$(\mathrm{a}) \Leftrightarrow(\mathrm{c})$. This follows from a duality due to Jannsen [8, Theorem 5.4] relating the $\Lambda$-modules $X^{\prime}$ and $Y$, together with a structure theorem for $Y$ due to Nguyen Quang Do (Corollary 14 of [11] or Theorem 4.4 of [9]).

$(\mathrm{c}) \Leftrightarrow(\mathrm{d})$. In proving the results cited in the previous case, one shows, in particular, that

$$
\operatorname{Tor}_{\Lambda}(Y) \simeq \operatorname{Ext}_{\Lambda}^{1}\left(X^{\prime}, \Lambda\right)
$$

[11, Theorem 9]. But $\operatorname{Ext}_{\Lambda}^{1}\left(X^{\prime}, \Lambda\right)$ is known to be the Pontryagin dual of $\lim _{F} X_{F}^{\prime}$ [11, Theorem 8]. The result then follows.

(c) $\Leftrightarrow(\mathrm{e})$. Grothendieck's local duality can be used to show that a torsion $\Lambda$-module is pseudo-null if and only if $\operatorname{Ext}_{\Lambda}^{1}$ vanishes [11, Lemma 6]. This implies, in particular, that $\operatorname{Ext}_{\Lambda}^{1}(X, \Lambda)$ and $\operatorname{Ext}_{\Lambda}^{1}\left(X^{\prime}, \Lambda\right)$ are isomorphic, yielding

$$
\operatorname{Tor}_{\Lambda}(Y) \simeq \operatorname{Ext}_{\Lambda}^{1}(X, \Lambda)
$$

as well. Theorem 4 then finishes the proof.

REMARK. Various forms of these equivalences have certainly appeared elsewhere. In [9], Lannuzel and Nguyen Quang Do prove the equivalence of (a), (c), and (e) under slightly different hypotheses. Namely, no restriction is made on the number of primes of $K$ dividing $p$, but rather it is assumed that all finite extensions of $K$ in $\widetilde{K}$ satisfy Leopoldt's conjecture. Formulation (c) has been used by McCallum [11] and this author [10] to verify Greenberg's conjecture for certain classes of cyclotomic fields.

The following theorem provides sufficient conditions for when the pseudonull conjecture for a number field $K$ implies the conjecture for a finite extension of $K$ in $\widetilde{K}$. We apply this to the cyclotomic tower in Section 4.

Theorem 6. Let $p \geq 5$ be a prime and suppose $\mu_{p}$ is contained in $K$. Suppose $K$ has a unique prime $\wp$ dividing $p$. Then, if $F \subset \widetilde{K}$ is a finite extension of $K$ satisfying

(1) $\wp$ is non-split in $F / K$,

(2) $\operatorname{dim}_{\mathbb{F}_{p}} H^{2}\left(\mathcal{G}_{F}, \mathbb{Z} / p \mathbb{Z}\right) \leq 1$,

(3) Leopoldt's conjecture,

then Greenberg's conjecture for K implies Greenberg's conjecture for F.

Proof. Let $K$ and $F$ be number fields satisfying the above hypotheses, and assume the pseudo-null conjecture holds for $K$. We apply the notation introduced in Section 2 to the field $F$ (so we have $\Omega_{F}, \mathcal{G}_{F}, M_{F}$, etc.). If the $\mathbb{F}_{p}$-dimension of $H^{2}\left(\mathcal{G}_{F}, \mathbb{Z} / p \mathbb{Z}\right)$ is 0 , then $\mathcal{G}_{F}$ is a free pro-p-group. A structure theorem for $Y$ due to Nguyen Quang Do [12, Proposition 1.7] then 
implies $\operatorname{Tor}_{\Lambda}(Y)=0$. Hence by formulation (c) of Theorem 5 Greenberg's conjecture holds for $F$.

If the $\mathbb{F}_{p}$-dimension of $H^{2}\left(\mathcal{G}_{F}, \mathbb{Z} / p \mathbb{Z}\right)$ is 1 , then such an $F$ satisfies the hypotheses of Theorem 3 and Lemma 1, and so Corollary 1 applies. Namely, Greenberg's pseudo-null conjecture will hold for $F$ provided $M_{F} \subset N_{\infty}$, and hence it will suffice to show the extension $M_{F} / \widetilde{F}$ is generated by $p$-power roots of $p$-units of $\widetilde{F}$.

We consider the field $F^{\text {cyc }}=F K^{\text {cyc }}$, the cyclotomic $\mathbb{Z}_{p}$-extension of $F$. By assumption, this field contains all $p$-power roots of unity. Recall the group $\mathcal{G}_{F}^{\text {ab }}=\operatorname{Gal}\left(M_{F} / F\right)$. The subgroup $\operatorname{Gal}\left(M_{F} / F^{\mathrm{cyc}}\right)$ has the same torsion subgroup (which is just $\operatorname{Gal}\left(M_{F} / \widetilde{F}\right)$ ) and $\mathbb{Z}_{p}$-rank 1 less. In particular, we have a non-canonical isomorphism

$$
\operatorname{Gal}\left(M_{F} / F^{\mathrm{cyc}}\right) \simeq \operatorname{Gal}\left(\widetilde{F} / F^{\mathrm{cyc}}\right) \times \operatorname{Gal}\left(M_{F} / \widetilde{F}\right) .
$$

We let $L$ denote the fixed field of the first factor (so $M_{F}=\widetilde{F} L$ ).

The Galois group $\operatorname{Gal}\left(L / F^{\text {cyc }}\right)$ is isomorphic to the torsion subgroup of $\mathcal{G}_{F}^{\text {ab }}$, and hence is a finite $p$-group. Since $F^{\text {cyc }}$ contains all $p$-power roots of unity, the extension $L / F^{\text {cyc }}$ is just a Kummer extension, generated by $p$-power roots of elements of $F^{\text {cyc: }}$

$$
L=F^{\mathrm{cyc}}\left(x_{1}^{1 / p^{m_{1}}}, x_{2}^{1 / p^{m_{2}}}, \ldots, x_{n}^{1 / p^{m_{n}}}\right) .
$$

Further, the ideals $\left(x_{i}\right)$ are $p^{m_{i}}$ th powers of ideals of $F^{\text {cyc }}$, say $\left(x_{i}\right)=\mathfrak{J}_{i}^{p^{m_{i}}}$. The extension $M_{F} / \widetilde{F}$ is also generated by the $x_{i}^{1 / p^{m_{i}}}$, and the ideals $\left(x_{i}\right)$ are the $p^{m_{i}}$ th powers of the ideals $\mathfrak{J}_{i}$ extended to $\widetilde{F}$. But here is the key: the ideal classes $\left[\widetilde{J}_{i}\right]$ become principal classes when extended to $\widetilde{F}$. This follows from the fact that $F^{\text {cyc }} \subset \widetilde{K}$ and, having assumed the pseudo-null conjecture holds for $K$ (using formulation (e) of Theorem 5), the fact that all ideal classes become principal in $\widetilde{K}$.

For a generator $x_{i}^{1 / p^{m_{i}}}$ of $M_{F} / \widetilde{F}$ we now know the ideal $\left(x_{i}\right)$ is the $p^{m_{i}}$ th power of a principal ideal, say

$$
\left(x_{i}\right)=\left(y_{i}\right)^{p^{m_{i}}} .
$$

The elements $x_{i}$ and $y_{i}^{p^{m_{i}}}$ must differ by a unit, say $x_{i}=u y_{i}^{p^{m_{i}}}$. But clearly, an extension generated by a $p^{m_{i}}$ th root of $x_{i}$ is also generated by a $p^{m_{i}}$ th root of $x_{i} /\left(y_{i}^{p^{m_{i}}}\right)=u$, and so the extension $M_{F} / \widetilde{F}$ is generated by $p$-power roots of units on $\widetilde{F}$. This implies $M_{F} \subset N_{\infty}$, which in turn, by Corollary 1 and Theorem 5, implies Greenberg's conjecture for $F$.

4. Cyclotomic fields. We fix $p$ a prime number and consider more closely the case of the cyclotomic fields $K=\mathbb{Q}\left(\zeta_{p^{n}}\right)$. Recall that the group $\mathcal{G}_{K}$ has a minimal presentation as a pro-p-group with $g$ generators and $s$ 
relations, where $g$ and $s$ are equal to the $\mathbb{F}_{p^{-}}$dimensions of $H^{1}\left(\mathcal{G}_{K}, \mathbb{Z} / p \mathbb{Z}\right)$ and $H^{2}\left(\mathcal{G}_{K}, \mathbb{Z} / p \mathbb{Z}\right)$ respectively.

Lemma 2. Let $p$ be a prime and let $K=\mathbb{Q}\left(\zeta_{p^{n}}\right)$ for some natural number $n$. Let $\alpha$ denote the $\mathbb{Z} / p \mathbb{Z}$-rank of the $p$-class group of $K$. Then

$$
g=\frac{p^{n}+p^{n-1}+2}{2}+\alpha, \quad s=\alpha .
$$

Proof. These computations are not new, and we give here just a sketch. Let $\Omega_{K}^{\prime}$ be the maximal $p$-ramified extension of $K$ with Galois group $\mathcal{G}_{K}^{\prime}$. Since $K$ contains the group $\mu_{p}$, and $\mathcal{G}_{K}$ is the maximal pro-p-quotient of $\mathcal{G}_{K}^{\prime}$, we have

$$
H^{i}\left(\mathcal{G}_{K}, \mathbb{Z} / p \mathbb{Z}\right) \simeq H^{i}\left(\mathcal{G}_{K}^{\prime}, \mu_{p}\right) .
$$

The $\mathbb{Z} / p \mathbb{Z}$-dimensions of the latter groups can be obtained by considering the sequence

$$
1 \rightarrow \mu_{p} \rightarrow \mathcal{O}_{\Omega_{K}^{\prime}}[1 / p]^{\times} \stackrel{p}{\longrightarrow} \mathcal{O}_{\Omega_{K}^{\prime}}[1 / p]^{\times} \rightarrow 1 .
$$

The $p$-power map on $\mathcal{O}_{\Omega_{K}^{\prime}}[1 / p]^{\times}$is surjective by the maximality of $\Omega_{K}^{\prime}$ over $K$ (since $p$ th roots of $p$-units generate $p$-ramified extensions). Taking cohomology of the sequence with respect to the Galois group $\mathcal{G}_{K}^{\prime}$ yields a long exact sequence which may be broken into the following pair of short exact sequences:

$$
\begin{aligned}
0 & \rightarrow \frac{\mathcal{O}_{K}[1 / p]^{\times}}{\left(\mathcal{O}_{K}[1 / p]^{\times}\right)^{p}} \rightarrow H^{1}\left(\mathcal{G}_{K}^{\prime}, \mu_{p}\right) \rightarrow C(K)[p] \rightarrow 0, \\
0 & \rightarrow \frac{C(K)}{p C(K)} \rightarrow H^{2}\left(\mathcal{G}_{K}^{\prime}, \mu_{p}\right) \rightarrow H^{2}\left(\mathcal{G}_{K}^{\prime}, \mathcal{O}_{\Omega_{K}^{\prime}}[1 / p]^{\times}\right)[p] \rightarrow 0,
\end{aligned}
$$

where $C(K)$ is the ideal class group of $K$. The group $H^{2}\left(\mathcal{G}_{K}^{\prime}, \mathcal{O}_{\Omega_{K}^{\prime}}[1 / p]^{\times}\right)$ injects into the Brauer group $B(K)$, and can be shown to be 0 by considering its behavior in the exact sequence

$$
0 \rightarrow B(K) \rightarrow \bigoplus_{v} B\left(K_{v}\right) \stackrel{\sum i n v}{\longrightarrow} \mathbb{Q} / \mathbb{Z} \rightarrow 0
$$

A simple dimension count then gives

$$
g=r_{2}+1+\alpha, \quad s=\alpha,
$$

where $r_{2}=\left(p^{n}+p^{n-1}\right) / 2$, as desired.

If $p$ is a regular prime, $\alpha=0$ for $\mathbb{Q}\left(\zeta_{p^{n}}\right), n \geq 0$. Hence $s=0$, implying $\operatorname{Tor}_{\Lambda}(Y)=0$, establishing Greenberg's conjecture for each field in the cyclotomic tower. 
The following corollary is an immediate consequence of Theorem 6 and Lemma 2.

COROllary 2. Let $p$ be an irregular prime. Let $n>0$ be such that $\mathbb{Q}\left(\zeta_{p^{n}}\right)$ has a cyclic p-class group. Then Greenberg's conjecture for $\mathbb{Q}\left(\zeta_{p}\right)$ implies Greenberg's conjecture for $\mathbb{Q}\left(\zeta_{p^{n}}\right)$.

Proof. In the notation of Theorem 6 , with $K$ as above, let $F=\mathbb{Q}\left(\zeta_{p^{n}}\right)$ for some positive integer $n$ satisfying the hypothesis. The field $K$ has a unique prime $\pi$ above $p$, and $\pi$ is totally ramified in $F / K$, and hence non-split. The dimension of $H^{2}\left(\mathcal{G}_{F}, \mathbb{Z} / p \mathbb{Z}\right)$ is less than or equal to 1 by our assumption of cyclic $p$-class groups. Since $F / \mathbb{Q}$ is abelian, implying Leopoldt's conjecture for $F$, the hypotheses of Theorem 6 are satisfied, as desired.

Finally, we prove Theorem 1 by providing a class of cyclotomic fields $\mathbb{Q}\left(\zeta_{p}\right)$, satisfying the hypotheses of Corollary 2, for which the pseudo-null conjecture is true. A similar class was first given by McCallum [11, Theorem 1]. He considered such fields with $p$-class group isomorphic to $\mathbb{Z} / p \mathbb{Z}$. We provide here a slight generalization of that class, allowing for cyclic $p$-class groups of arbitrary $p$-power order, as well as apply Corollary 2 to extend the conjecture to all fields in the cyclotomic $\mathbb{Z}_{p}$-tower. We restate Theorem 1 here.

Theorem 7. Suppose $K=\mathbb{Q}\left(\zeta_{p}\right)$ satisfies the following conditions:

(1) Vandiver's conjecture.

(2) $\lambda_{p}=1$.

(3) $v_{p}\left(\left|(U / \bar{E})\left[p^{\infty}\right]\right|\right) \leq v_{p}(|A(K)|)$.

Then for all $n \geq 1$ the pseudo-null conjecture holds for $\mathbb{Q}\left(\zeta_{p^{n}}\right)$.

REMARK 1 . Condition (2) is heuristically true for approximately $75 \%$ of all irregular primes and experimentally true for $75 \%$ of the irregular primes up to 12 million, according to [2] (for these primes, $\lambda_{p}$ is just the index of irregularity of $p$ ).

Remark 2. Letting $K_{n}=\mathbb{Q}\left(\zeta_{p^{n+1}}\right)$ and $A_{n}=A\left(K_{n}\right)$, the hypotheses of Vandiver's conjecture and $\lambda_{p}=1$ imply

$$
A_{n} \simeq X /\left((1+T)^{p^{n}}-1\right) X,
$$

where $X=\mathbb{Z}_{p}[[T]] /\left(T+p^{a}\right)$ (see Theorem 10.16 and Proposition 13.22 of [14]). In particular this yields isomorphisms

$$
A_{n} \simeq \mathbb{Z} / p^{a+n} \mathbb{Z}
$$

for all $n \geq 0$, and so (3) is a condition on cyclic groups of $p$-power order.

REMARK 3. Since $A(K)$ is cyclic, there is only one Bernoulli number $B_{i}, 2 \leq i \leq p-3$, divisible by $p$. If $B_{p-j}$ denotes this term (so $\varepsilon_{j} A(K)$ is the non-trivial term of the idempotent decomposition of $A(K))$, then 
$L_{p}\left(s, \omega^{1-j}\right)$ is the only non-trivial $p$-adic $L$-function attached to $K$. It follows from Theorem 8.25 of [14] that

$$
(U / \bar{E})\left[p^{\infty}\right] \simeq \mathbb{Z} / p^{m} \mathbb{Z}
$$

where $m=v_{p}\left(L_{p}\left(1, \omega^{1-j}\right)\right)$. This valuation may be computed in terms of the characteristic power series $f(T)$ of $\lim _{n} A\left(K_{n}\right)$. Under the assumption $\lambda_{p}=1$ this power series has the form $f\left(\overleftarrow{T)}=\left(T+c p^{a}\right) u\right.$, where $u$ is a unit, $p^{a}$ is the order of the cyclic group $A(K)$, and

$$
f\left((1+p)^{s}-1\right)=L_{p}\left(s, \omega^{1-j}\right) .
$$

So the valuation of $L_{p}$ at $s=1$ equals the valuation of $f(p)=\left(p+c p^{a}\right) u$.

If $a>1$, then $v_{p}(f(p))=1$, and condition (3) is satisfied. If, on the other hand, $a=1$, then $v_{p}(f(p))$ depends on the value of $c(\bmod p)$. The valuation will again be 1 provided $c \not \equiv-1(\bmod p)$. This congruence has been checked for $p<4000$ in [6], although tables are only given for $p<400$ and $3600<p<4000$. For these values the congruence condition is satisfied.

Suppose $K=\mathbb{Q}\left(\zeta_{p}\right)$ satisfies (1)-(3) above. Since $A(K)$ is cyclic, say of order $p^{a}$, the group $\mathcal{G}$ is a one-relator group and Lemma 1 applies. We will utilize this lemma to show $\operatorname{Tor}_{\Lambda}(Y)=0$. In light of Corollary 1, it suffices to show $M_{K} \subset N_{\infty}$, and so we consider the structure of $\mathcal{G}_{K}^{\text {ab }}$ in more detail.

Lemma 3. Suppose $K$ satisfies hypotheses (1) and (2) of Theorem 7. Then the torsion subgroup of $\mathcal{G}_{K}^{\mathrm{ab}}$ is cyclic.

Proof. Let $J_{K}$ denote the idele group of $K$, with $K^{\times}$embedded diagonally. Let $U$ be the subgroup of ideles which are units at $\pi$ (the prime of $K$ above $p$ ) and 1 elsewhere, and let $U^{\prime}$ be the subgroup of ideles which are 1 at $\pi$ and units elsewhere. Class field theory gives an isomorphism

$$
\mathcal{G}_{K}^{\mathrm{ab}} \simeq \text { pro-p-completion of } J_{K} /\left(\overline{K^{\times} U^{\prime}}\right),
$$

where the overline denotes the closure.

If we let $\bar{E}$ denote the closure of the embedding of the units of $K$ in $U$, then in fact we have an exact sequence

$$
0 \rightarrow U_{1} / \bar{E}_{1} \rightarrow \mathcal{G}_{K}^{\mathrm{ab}} \rightarrow A(K) \rightarrow 0,
$$

where the subscript 1 indicates we are taking units congruent to 1 modulo $\pi$. Since $U_{1}$ has $\mathbb{Z}_{p}$-rank $[K: \mathbb{Q}]=p-1$ and $\bar{E}_{1}$ has $\mathbb{Z}_{p}$-rank $(p-3) / 2$ (by

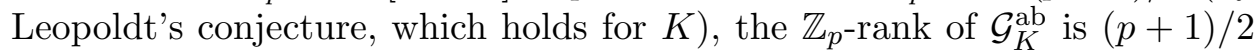
$\left(p \neq 2\right.$ by the assumption $\left.\lambda_{p}=1\right)$.

We claim that the torsion in $\mathcal{G}_{K}^{\text {ab }}$ comes from $U_{1} / \bar{E}_{1}$, and show this by considering an idele $\left(a_{v}\right)$ whose image in $\mathcal{G}_{K}^{\text {ab }}$ is a torsion element, say of order $p^{m}$. So

$$
\left(a_{v}\right)^{p^{m}} \in \overline{K^{\times} U^{\prime}}
$$


say $\left(a_{v}\right)^{p^{m}}=\alpha\left(u_{v}\right)$ (where we abuse notation writing $\alpha$ for both the element of $K^{\times}$as well as its diagonal image in $J_{K}$ ). This implies $\alpha$ is a $p^{m}$ th power in $K_{\pi}$, the $\pi$-adic completion of $K$. Let $\mathfrak{a}$ then be the ideal of $K$ such that $\mathfrak{a}^{p^{m}}=(\alpha)$. We want to show the class of $\mathfrak{a}$ is principal.

Let $K_{m-1}=\mathbb{Q}\left(\zeta_{p^{m}}\right)$, so $K_{m-1}\left(\alpha^{1 / p^{m}}\right)$ is an unramified extension. Since the class of $\mathfrak{a}$ lies in $A(K)^{-}$(by Vandiver's conjecture), the Kummer pairing implies the Galois group of $K_{m-1}\left(\alpha^{1 / p^{m}}\right) / K_{m-1}$ is trivial. Hence $\alpha$ must be a $p^{m}$ th power in $K_{m-1}$ as well, which means the ideal class of $\mathfrak{a}$ is principal when extended to $K_{m-1}$ (represented by a principal ideal generated by a $p^{m}$ th root of $\left.\alpha\right)$. But the map from $A(K)$ to $A\left(K_{m-1}\right)$ is injective [14, Proposition 13.26], and so $\mathfrak{a}$ must have represented a principal class in $A(K)$ as well. Hence the torsion in $\mathcal{G}_{K}^{\text {ab }}$ maps to 0 in $A(K)$.

We now just need to determine the torsion subgroup of $U_{1} / \bar{E}_{1}$. We may consider each factor of the idempotent decomposition separately. Since $\varepsilon_{i} E_{1}=0$ for $i=0$ and for $i$ odd, and each $\varepsilon_{i} U_{1} \simeq \mathbb{Z}_{p}$, we obtain

$$
U_{1} / \bar{E}_{1} \simeq\left(\mathbb{Z}_{p}\right)^{(p+1) / 2} \oplus \bigoplus_{i \text { even }} \varepsilon_{i} U_{1} / \varepsilon_{i} \bar{E}_{1} .
$$

For even $i$ the terms $\varepsilon_{i} U_{1} / \varepsilon_{i} \bar{E}_{1}$ are equal to $\varepsilon_{i} U_{1}^{+} / \varepsilon_{i} \bar{E}_{1}^{+}$, where the superscript + indicates we are looking at units in the local subfield fixed by the automorphism of order 2. Vandiver's conjecture implies the cyclotomic units $C_{1}^{+}$have index prime to $p$ in $E_{1}^{+}$[14, Theorem 8.2], and so it suffices to consider the quotients $\varepsilon_{i} U_{1}^{+} / \varepsilon_{i} \bar{C}_{1}^{+}$. But Theorem 8.25 of [14] states

$$
\left[\varepsilon_{i} U_{1}^{+}: \varepsilon_{i} \bar{C}_{1}^{+}\right]=p^{v_{p}\left(L_{p}\left(1, \omega^{i}\right)\right)} .
$$

Since $A(K)$ is cyclic there is only one non-trivial $L_{p}\left(s, \omega^{i}\right)$, and hence only one cyclic factor, say of order $p^{m}$, in the torsion subgroup of $U_{1} / \bar{E}_{1}$.

Proof of Theorem $\%$. The field $\widetilde{K}$ is in fact the fixed field of the torsion subgroup of $\mathcal{G}_{K}^{\mathrm{ab}}$, and so the extension $M_{K} / \widetilde{K}$ is a Kummer extension with $\operatorname{Gal}\left(M_{K} / \widetilde{K}\right) \simeq \mathbb{Z} / p^{m} \mathbb{Z}$. With $A(K) \simeq \mathbb{Z} / p^{a} \mathbb{Z}$, condition (3) of the theorem just states $m \leq a$.

To show that $M_{K}$ is contained in $N_{\infty}$, we need to show that $M_{K} / \widetilde{K}$ is generated by a $p$ th power root of a unit of $\widetilde{K}$. The argument, as in the proof of Theorem 6 , is reduced to a capitulation problem.

Consider the extension $M_{K} / K_{m-1}$. There is a non-canonical isomorphism

$$
\operatorname{Gal}\left(M_{K} / K_{m-1}\right) \simeq \operatorname{Gal}\left(\widetilde{K} / K_{m-1}\right) \times \operatorname{Gal}\left(M_{K} / \widetilde{K}\right) .
$$

We let $L$ denote the fixed field of the first factor. The extension $L / K_{m-1}$ is a Kummer extension, and we may write

$$
L=K_{m-1}\left(x^{1 / p^{m}}\right)
$$


for some $x$ in $K_{m-1}$, the ideal $(x)$ being of the form $(x)=\mathfrak{J}^{p^{m}} P$, where $P$ is the principal ideal of $K_{m-1}$ lying above $p$.

Since, in particular, $\mathfrak{J}$ represents a class of order dividing $p^{m}$ in $A\left(K_{m-1}\right)$, condition (3) implies that the class of $\mathfrak{J}$ is an extension of a class from $A(K)$ (recall that the map $A(K) \rightarrow A\left(K_{m-1}\right)$ is just an injection $\mathbb{Z} / p^{a} \mathbb{Z} \hookrightarrow$ $\left.\mathbb{Z} / p^{a+m-1} \mathbb{Z}\right)$. We let $\mathfrak{A}$ be a representative ideal of the class that extends to the class of $\mathfrak{J}$.

Since the $p$-Hilbert class field of $K$ is contained in $\widetilde{K}$, the class of $\mathfrak{A}$, and therefore $\mathfrak{J}$, becomes principal in $\widetilde{K}$. The extension $M_{K} / \widetilde{K}$ is also generated by a $p^{m}$ th root of $x$, and the ideal $(x)$ in $\widetilde{K}$ is now the $p^{m}$ th power of a principal ideal,

$$
(x)=(y)^{p^{m}} \text {. }
$$

The elements $x$ and $y^{p^{m}}$ then differ by a unit, i.e. $x=u y^{p^{m}}$. But clearly the extension $M_{K}$ is also generated by the $p^{m}$ th root of $x / y^{p^{m}}=u$, and so the field $M_{K}$ is contained in $N_{\infty}$.

\section{References}

[1] W. Bruns and J. Herzog, Cohen-Macaulay Rings, Cambridge Stud. Adv. Math. 39, Cambridge Univ. Press, Cambridge, 1993.

[2] J. Buhler, R. Crandall, R. Ernvall, T. Metsankyla, and M. A. Shokrollahi, Irregular primes and cyclotomic invariants to 12 million, J. Symbolic Comput. 31 (2001), 89-96.

[3] R. Greenberg, The Iwasawa invariants of $\Gamma$-extensions of a fixed number field, Amer. J. Math. 95 (1973), 204-214.

[4] -, On the structure of certain Galois groups, Invent. Math. 47 (1978), 85-99.

[5] —, Iwasawa theory — past and present, in: Class Field Theory-Its Centenary and Prospect (Tokyo, 1998), Adv. Stud. Pure Math. 30, Math. Soc. Japan, Tokyo, 2001, $335-385$.

[6] K. Iwasawa and C. Sims, Computation of invariants in the theory of cyclotomic fields, J. Math. Soc. Japan 18 (1965), 86-96.

[7] U. Jannsen, On the structure of Galois groups as Galois modules, in: Number Theory Noordwijkerhout 1983, Lecture Notes in Math. 1068, Springer, Berlin, 1984, $109-126$.

[8] - Iwasawa modules up to isomorphism, in: Algebraic Number Theory, Adv. Stud. Pure Math. 17, Academic Press, Orlando, 1989, 171-207.

[9] A. Lannuzel et T. Nguyen Quang Do, Conjectures de Greenberg et extensions prop-libres d'un corps de nombres, Manuscripta Math. 102 (2000), 187-209.

[10] D. Marshall, Galois groups and Greenberg's conjecture, Ph.D. thesis, Univ. of Arizona, August 2000.

[11] W. McCallum, Greenberg's conjecture and units in multiple $\mathbb{Z}_{p}$-extensions, Amer. J. Math. 123 (2001), 909-930.

[12] T. Nguyen Quang Do, Formations de classes et modules d'Iwasawa, in: Number Theory Noordwijkerhout 1983, Lecture Notes in Math. 1068, Springer, Berlin, 1984, $167-185$. 
[13] J.-P. Serre, Galois Cohomology, Springer, Berlin, 1997.

[14] L. Washington, Introduction to Cyclotomic Fields, 2nd ed., Grad. Texts in Math. 83, Springer, New York, 1997.

[15] M. Yamagishi, On free pro-p-extensions of algebraic number fields, Sūrikaisekikenkyūsho Kōkyūroku 884 (1994), 172-177.

Department of Mathematics

University of Texas at Austin

Austin, TX 78712, U.S.A.

E-mail: marshall@math.utexas.edu

Received on 1.10.2001

and in revised form on 5.7.2003 